DOI 10.5216/ia.v45i3.67009

\title{
UNA DÉCADA EVALUANDO LA CALIDAD DEL POSGRADO EN MÉXICO (2008-2018)
}

\author{
Josefina PATIÑo SALCEDA \\ Universidad Pedagógica Nacional (UPN), Ciudad de México, México \\ ARMANDO AlCÁNTARA \\ Universidad Nacional Autónoma de México (UNAM), Ciudad de México, México
}

\begin{abstract}
Resumo: En este artículo analizamos si el posgrado en México se ha desarrollado de acuerdo con los objetivos planteados en los programas de política nacional durante 2008-2018. A través del método de investigación evaluativa contrastamos los objetivos de la política con el comportamiento (incremento y distribución) de las instituciones, matrícula y programas acreditados por su calidad, distinguiendo al sector público y privado. Los resultados muestran que los objetivos de las políticas buscaban resolver: la insuficiencia de recursos humanos para la investigación y el bajo porcentaje de posgrados de calidad. Sin embargo, el análisis de los datos estadísticos muestra que dichos objetivos no tuvieron efecto en el desarrollo del posgrado, dando como resultado un incremento acelerado del sector privado en programas sin acreditación de calidad.
\end{abstract}

Palavras-chave: Posgrado. Evaluación. Calidad. México.

\section{INTRODUCCIÓN}

En el mundo actual el posgrado es nivel máximo de estudios que ofrecen las instituciones de educación superior (IES), y se espera que sus graduados sean los actores principales de las economías del conocimiento. La expectativa es que los posgraduados se dediquen a actividades de investigación e innovación para contribuir al incremento de la productividad económica, el bienestar social y la reducción de la pobreza. Bajo estos supuestos, el número de doctores aumentó 40\% entre1998 y 2006 en los países miembros de la Organización para la Cooperación y el Desarrollo Económicos (OCDE) (AURIOL, 2010). Por lo anterior, vale la pena preguntarse: ¿Cuáles son los planes de México respecto al posgrado? ¿Está vinculado su desarrollo a los objetivos de los programas gubernamentales? En este artículo buscamos responder a estas dos preguntas. 
Nuestro principal supuesto es que las políticas se han enfocado principalmente al sector público del posgrado, mientras que el sector privado o particular se regula bajo la lógica de la oferta y la demanda. Para comprobarlo recurrimos a la investigación evaluativa cuyo objetivo es valorar la eficacia o éxito de un programa de acuerdo con criterios específicos, con el fin de tomar decisiones presumiblemente óptimas de la situación (CABRERA, 1987; SANDÍN, 2003). Este método también ha sido utilizado para evaluar sistemas educativos, por lo que es el método más conveniente para nuestros propósitos. La investigación evaluativa utiliza las metodologías de la investigación social para juzgar y mejorar la planificación, la verificación, la efectividad y la eficacia de programas sociales de salud, educación, bienestar comunitario y otros (ROSSI y FREEMAN, 1993; SANDÍN, 2003). Es en este sentido que buscamos hacer una aportación para mejorar el estado actual del posgrado en el país.

Para lograr el objetivo y poner a prueba nuestro supuesto analizamos un periodo de 10 años, pues consideramos que es un tiempo suficiente para los propósitos de esta investigación. Primero, revisamos los programas de política científica y de educación para identificar si existió alguna intención específica para la mejora del posgrado y la evaluación de su calidad. Posteriormente, analizamos el aumento en el número de instituciones, la matrícula y los programas acreditados por su calidad, considerando incrementos porcentuales por sector de sostenimiento (público, particular) y por nivel educativo. Finalmente, discutimos si el comportamiento de la matrícula y los programas acreditados muestran algún efecto relacionado con los objetivos, estrategias y acciones establecidos en los planes y programas de política.

\section{LOS PROGRAMAS DE POLÍTICA CIENTÍFICA Y EDUCATIVA EN MÉXICO 2008-2018}

En este apartado analizamos los programas de política científica a través del Programa Especial de Ciencia y Tecnología (PECyT) 2008-2012 y el Programa Especial de Ciencia, Tecnología e Innovación (Peciti) 2014-2018 y los programas de política educativa mediante el Programa Sectorial de Educación (PSE) 2007-2012 y el PSE 2013-2018. Con este análisis mostramos los ideales respecto al subsistema de posgrado, sus principales problemas identificados en los diagnósticos y los objetivos trazados por el gobierno mexicano a través de estos documentos.

¿Qué se espera del posgrado? En los programas de ciencia y tecnología se establece una relación directa entre el fortalecimiento de la ciencia y el crecimiento económico sustentable, la solución de las necesidades del país y la mejora de vida de la población; por lo que se reconoce la necesidad de incrementar la formación de recursos humanos, entendiendo que este aspecto es indispensable para fortalecer el sistema. Se afirma que: 
SALCEDA, J. P.; ALCÁNTARA, A.

La evidencia empírica muestra que el impulso a la generación, absorción y consolidación de capital humano altamente calificado para llevar a cabo funciones de investigación y la construcción de infraestructura moderna dedicada a este mismo fin, son elementos determinantes para impulsar el desarrollo de un sistema nacional de CTI equilibrado y con alto potencial para construir una economía del conocimiento (CONACYT, 2014, p. 22).

¿Cuál es el diagnóstico respecto a la formación de recursos humanos? Tanto en los programas de ciencia y tecnología como en el PSE 2013-2018 se hace evidente la insuficiencia de doctores que realicen investigación de alta calidad, asimismo se muestra que el indicador internacional de investigadores por cada 1,000 miembros de la Población Económicamente Activa (PEA) se encuentra por debajo del promedio de los países de la OCDE:

La comparación es muy desfavorable para México, aunque ha mejorado, apenas alcanzó la cifra de 0.98 en 2012; habría que multiplicarla por un factor de siete para acercarse al promedio de los países de la OCDE (PSE 2013, p. 33).

\section{EVALUANDO LA CALIDAD DE LOS POSGRADOS}

Un instrumento de política científica y educativa de suma importancia se refiere a los programas de evaluación de la calidad del posgrado que iniciaron su operación desde 1991. En el PECyT 2008-2012 se reconoce ampliamente la importancia del Programa Nacional de Posgrados de Calidad; sin embargo, no señala la urgencia de incrementar el número de programas con registro de calidad. En cambio, tanto en el PSE 2013-2018 como en el Peciti 2014-2018 se reconoce que la calidad del posgrado es todavía uno de los mayores retos debido a que del total de programas que existen en el país, menos de la cuarta parte logran obtener el reconocimiento de su calidad:

Durante el periodo 2006-2012, el padrón se incrementó más de dos veces, lo que es un avance significativo para el SNCTI [Sistema Nacional de Ciencia, Tecnología e Innovación]. Sin embargo, únicamente representa $19 \%$ de un universo mucho mayor de programas registrados por la SEP a nivel nacional (en 2012 había en México 8,317 programas de posgrado escolarizados) (CONACYT, 2014, p. 23).

A pesar de que en el PECyT 2008-2012 no se expresa de manera explícita la necesidad de incrementar la calidad del posgrado del sector privado, señala la necesidad de reestructurar las políticas públicas en la materia y de mejorar las bases normativas y legales para lograr la educación de calidad y la incorporación de jóvenes a la investigación: 
Una década evaluando la calidad del posgrado...

\begin{abstract}
La reestructuración y el fortalecimiento de la política pública en materia de conocimiento e innovación resultan fundamentales para crear condiciones que permitan alcanzar mayores niveles de crecimiento de la economía. Son procesos complejos que incluyen el establecimiento de bases normativas y legales; educación de calidad e incorporación de jóvenes en las tareas de investigación; generación y aplicación del conocimiento e innovación en las actividades industriales y de servicios (CONACYT, 2008, p. 3).
\end{abstract}

¿Cuáles fueron los objetivos planteados en los programas de ciencia, tecnología e innovación en el periodo bajo estudio? Tanto en el PECyT 2008-2012 como en el Peciti 20142018 se estableció un objetivo destinado a la formación de recursos humanos. En 2008 este rubro tuvo entre sus propósitos:

Evaluar la aplicación de los recursos públicos que se invertirán en la formación de recursos humanos de alta calidad (científicos y tecnólogos), y en las tareas de investigación científica, desarrollo tecnológico e innovación, de tal manera que se canalicen a áreas prioritarias para el país, con el objetivo de que tengan el mayor impacto social y económico posible (CONACYT, 2008, p. 46).

Por su parte, en el plan de 2014 los objetivos se enfocaron a la formación y fortalecimiento del capital humano de alto nivel, así como al impulso del desarrollo de las vocaciones y capacidades de CTI locales, con el fin de fortalecer el desarrollo regional sustentable e incluyente (CONACYT, 2014).

Con la información anterior podemos ver que en ambos programas fue importante la formación de recursos humanos. Sin embargo, el objetivo de 2008 lleva implícito que se refiere solo al sector privado del posgrado, dado que la inversión en la formación de recursos humanos se otorga a estudiantes inscritos en programas que obtienen el reconocimiento de calidad por parte del PNPC, y como se verá más adelante, el Padrón del PNPC está integrado en un 94 por ciento por programas adscritos a instituciones públicas, mientras que sólo el 6 por ciento pertenecen a una institución privada (SÁNCHEZ, 2017). En cambio, los objetivos de 2014 se refieren a la formación de capital humano de alto nivel, así como al desarrollo de las vocaciones y capacidades científicas y tecnológicas sin centrarse en alguno de los dos sectores.

Tanto en el PSE 2007-2012 como en el de 2013-2018 los gobiernos pusieron énfasis en la calidad de la educación. En 2007, a través del "Objetivo 1. Elevar la calidad de la educación para que los estudiantes mejoren su nivel de logro educativo, cuenten con medios para tener acceso a un mayor bienestar y contribuyan al desarrollo nacional" (SEP, 2007, p. 11). En 2013, a través del "Objetivo 2. Fortalecer la calidad y pertinencia de la educación media superior, superior y formación para el trabajo, a fin de que contribuyan al desarrollo de 
SALCEDA, J. P.; ALCÁNTARA, A.

México" (SEP, 20013, p. 48). Derivado de estos se establecieron estrategias y acciones que vale la pena revisar (ver Tabla 1).

En ambos programas se hace evidente la preocupación por incrementar el número de programas de posgrado con reconocimiento de su calidad. No obstante, en el PSE 20072012, en la estrategia 1.16 se enfatiza la preocupación por garantizar la calidad de los programas considerando ambos sectores de sostenimiento. En cambio, en la estrategia 1.17 se hace referencia de manera específica a las instituciones del sector privado. Las acciones planteadas se refieren a preocupaciones muy concretas: a) la revisión de los criterios para otorgar el Reconocimiento de Validez Oficial de Estudios (RVOE); b) la aplicación homogénea de estos criterios; c) el fortalecimiento de la capacidad de las autoridades para evaluar los programas; y d) la garantía de la calidad de los programas de IES privadas a través de procesos de evaluación externa.

A diferencia del PSE 2007-2012, en el PSE 2013-2018 se establecen acciones sin referirse a un sector de sostenimiento específico, sin embargo, se menciona que se buscará trabajar de manera coordinada con el Conacyt para incrementar la oferta en el PNPC (ver Tabla 1).

Tabla 1. Estrategias y acciones para el posgrado en los PSE 2007-2012 y 2013-2018

\begin{tabular}{|c|c|c|}
\hline Programas & Estrategias & Acciones \\
\hline \multirow{5}{*}{$\begin{array}{c}\text { PSE 2007- } \\
2012\end{array}$} & $\begin{array}{l}\text { 1.16 Contribuir a extender y arraigar } \\
\text { una cultura de la planeación, de la } \\
\text { evaluación y de la mejora continua de la } \\
\text { calidad educativa en las instituciones de } \\
\text { educación superior, tanto públicas } \\
\text { como particulares. }\end{array}$ & $\begin{array}{l}\text { Fortalecer y ampliar la cobertura de los programas dirigidos } \\
\text { a mejorar la calidad y pertinencia de los programas de } \\
\text { posgrado. }\end{array}$ \\
\hline & \multirow{4}{*}{$\begin{array}{l}1.17 \text { Garantizar que los programas que } \\
\text { ofrecen las instituciones de educación } \\
\text { superior particulares reúnan los } \\
\text { requisitos de calidad. }\end{array}$} & $\begin{array}{l}\text { Revisar las reglas y perfeccionar los criterios que regulan el } \\
\text { otorgamiento del Reconocimiento de Validez Oficial de } \\
\text { Estudios (RVOE) de tipo superior, con el concurso de los } \\
\text { gobiernos tanto federal como estatales y, en su caso, de las } \\
\text { IES. }\end{array}$ \\
\hline & & $\begin{array}{l}\text { Establecer convenios con las entidades federativas con miras } \\
\text { a aplicar criterios con una base homogénea entre la } \\
\text { Federación y las entidades para el otorgamiento de los } \\
\text { RVOE. }\end{array}$ \\
\hline & & $\begin{array}{l}\text { Fortalecer la capacidad técnica de las instancias del } \\
\text { Gobierno Federal y la de los gobiernos de las entidades } \\
\text { federativas, encargadas de analizar las solicitudes y otorgar } \\
\text { los RVOE, junto con la supervisión correspondiente. }\end{array}$ \\
\hline & & $\begin{array}{l}\text { Impulsar programas para que las instituciones particulares } \\
\text { con RVOE se involucren en los procesos de evaluación } \\
\text { externa, así como de aseguramiento de la calidad de los } \\
\text { servicios educativos que ofrecen (SEP, 2007, p. 28). }\end{array}$ \\
\hline
\end{tabular}




\begin{tabular}{|c|c|c|}
\hline $\begin{array}{l}\text { PSE 2013- } \\
2018\end{array}$ & $\begin{array}{l}\text { 2.4. Fomentar la investigación } \\
\text { científica y tecnológica y promover la } \\
\text { generación y divulgación de } \\
\text { conocimiento de impacto para el } \\
\text { desarrollo del país. }\end{array}$ & $\begin{array}{l}\text { Apoyar el crecimiento de la oferta de posgrado para la } \\
\text { formación de personas altamente calificadas en las áreas } \\
\text { que el país requiere. } \\
\text { Trabajar coordinadamente con el CONACYT para } \\
\text { incrementar la oferta en el Programa Nacional de } \\
\text { Posgrados de Calidad (PNPC). } \\
\text { Fortalecer las capacidades de investigación en las } \\
\text { instituciones de educación superior en áreas prioritarias del } \\
\text { país. } \\
\text { Incrementar el número y el nivel de los investigadores de } \\
\text { las instituciones de educación superior en el Sistema } \\
\text { Nacional de Investigadores. } \\
\text { Alentar la participación de estudiantes en actividades de } \\
\text { investigación (SEP, 20013, p. 48). }\end{array}$ \\
\hline
\end{tabular}

Fuente: Elaboración propia con base en los Programas Sectoriales de Educación 2007-2012 y 2013-2018 (SEP, 2007; 2014).

En síntesis, el PECyT 2008-2012 en sus objetivos se refiere de manera implícita al sector público del posgrado; no obstante, en un apartado del diagnóstico señala la necesidad de reestructurar la política pública haciendo alusión a la educación de calidad y a los jóvenes investigadores. El PSE 2007-2012 no presenta diagnóstico, pero su objetivo se centra en ambos sectores del posgrado, destacando de manera enfática la solución a los problemas relacionados con las instituciones particulares y su calidad. En cambio, los programas del periodo 2013-2018 en el diagnóstico se refieren a los dos sectores, haciendo énfasis en la necesidad de incrementar los posgrados de calidad, mientras que en los objetivos se refieren al posgrado de manera general.

\section{INCREMENTO DE LA MATRÍCULA, INSTITUCIONES Y PROGRAMAS DE POSGRADO DE CALIDAD 2008-2018}

En este apartado exponemos el marco normativo bajo el cual se rigen los estudios de posgrado en México con la intención de ofrecer un contexto que nos permita comprender las formas en las que se han incrementado las instituciones, la matrícula y los programas de posgrados acreditados por su calidad.

El posgrado (especialización, maestría y doctorado) se ofrece en un conjunto diverso de IES. De acuerdo con la caracterización que hace Javier Mendoza, esta diversidad de instituciones se refiere a su denominación, personalidad jurídica, tamaño, perfil académico y funciones, áreas del conocimiento en las que se ofertan los programas, extensión territorial; calidad; personal académico y su finalidad como establecimiento (Mendoza, 2018). Respecto a la autorización para impartir programas de tipo superior, el artículo tercero de la Constitución Política de los Estados Unidos Mexicanos en su fracción V establece que "el Estado proveerá y atenderá todos los tipos y modalidades educativos, incluyendo la educación inicial y la superior". En la fracción VI se establece el Reconocimiento de Validez Oficial de Estudios (RVOE), que es la autorización requerida para las instituciones de educación 
SALCEDA, J. P.; ALCÁNTARA, A.

superior particulares y en la fracción VII se reconoce la autonomía que por ley se ha otorgado a las universidades públicas (MENDOZA, 2018). Así, la Secretaría de Educación Pública (SEP) es la responsable de revisar y autorizar a través del REVOE los programas de tipo superior propuestos por las instituciones particulares; en cambio las instituciones públicas tienen sus propios procesos internos para la autorización de sus programas.

\section{LA MATRÍCULA DE POSGRADO POR RÉGIMEN DE SOSTENIMIENTO}

Existen datos que demuestran un fuerte incremento de matrícula en todo el sistema educativo mexicano entre los ciclos de 1970-1971 y 2002-2003. La matrícula de educación básica se duplicó pasando de 10 millones 750 mil 545 estudiantes a 24 millones 153 mil 164; la de educación media pasó de 369 mil 299 a 3 millones 275 mil 292 estudiantes; la de licenciatura pasó de 252 mil 236 a 1millón 931 mil 631 estudiantes (SILAS, 2005).

El incremento acelerado del sistema requirió de la incorporación de docentes a las instituciones de educación media superior y superior. Esta situación evidenció dos necesidades: a) la formación pedagógica para los docentes de nivel bachillerato; y b) la profesionalización para la docencia y la investigación. Estos dos factores fueron decisivos para que se incrementara la demanda de estudios de posgrado (WUEST y JIMÉNEZ, 1990).

El análisis de la matrícula de posgrado por régimen de sostenimiento (público o privado/particular), permitió identificar dos momentos en su expansión. El primero ocurrió en la década de los 70, pues se pasó de 226 programas en 1970 (50 de especialidad, 124 de maestría y 52 de doctorado) a 1,240 en 1980 (299 de especialidad, 807 maestrías y 134 de doctorado) (WUEST y JIMÉNEZ, 1990). Esta expansión fue soportada principalmente por el sector público, ya que en 1970 la participación del sector privado era apenas del 17 por ciento de la matrícula total.

El segundo momento de expansión ocurrió en los 90, cuando el sector particular incrementó notablemente su participación en la atención de la matrícula, pasando de 22 por ciento en 1990 a 40 por ciento en el año 2000 (PATIÑO, 2013). Esto significa que la expansión acelerada de la matrícula de posgrado en el sector particular ocurrió durante esta década. Ya para el ciclo 2008-2009, el sector particular atendía el 49 por ciento de la matrícula de posgrado, finalizando la década con poco más de 60 por ciento. Esto significa que este sector ha incrementado su participación con aproximadamente 10 puntos porcentuales por década en los últimos 20 años. En la Tabla 2 se puede ver con precisión el incremento en la participación del sector particular durante 2008-2018.

Tabla 2. Matrícula de posgrado por nivel y régimen de sostenimiento

\begin{tabular}{|c|c|c|c|c|c|c|c|c|}
\hline Ciclo & Régimen & $\mathbf{E}$ & $\boldsymbol{\%}$ & $\mathbf{M}$ & $\boldsymbol{\%}$ & $\mathbf{D}$ & $\boldsymbol{\%}$ & Total \\
\hline \multirow{3}{*}{$\mathbf{2 0 0 8 - 2 0 0 9}$} & Público & 26,348 & $66 \%$ & 54,864 & $43 \%$ & 13,676 & $74 \%$ & 94,888 \\
\cline { 2 - 9 } & Particular & 13,356 & $34 \%$ & 72,328 & $57 \%$ & 4,854 & $26 \%$ & 90,538 \\
\cline { 2 - 9 } & Total & 39,704 & $100 \%$ & 127,192 & $100 \%$ & 18,530 & $100 \%$ & 185,426 \\
\hline
\end{tabular}


Una década evaluando la calidad del posgrado...

Dossiê 660

\begin{tabular}{|c|c|c|c|c|c|c|c|c|}
\hline \multirow{3}{*}{ 2009-2010 } & Público & 28,080 & $71 \%$ & 58,582 & $43 \%$ & 15,123 & $72 \%$ & 101,785 \\
\hline & Particular & 11,731 & $29 \%$ & 77,134 & $57 \%$ & 5,747 & $28 \%$ & 94,612 \\
\hline & Total & 39,811 & $100 \%$ & 135,716 & $100 \%$ & 20,870 & $100 \%$ & 196,397 \\
\hline \multirow{3}{*}{$2010-2011$} & Público & 27,895 & $69 \%$ & 59,551 & $41 \%$ & 15,852 & $69 \%$ & 103,298 \\
\hline & Particular & 12,665 & $31 \%$ & 84,992 & $59 \%$ & 7,270 & $31 \%$ & 104,927 \\
\hline & Total & 40,560 & $100 \%$ & 144,543 & $100 \%$ & 23,122 & $100 \%$ & 208,225 \\
\hline \multirow{3}{*}{ 2011-2012 } & Público & 29,730 & $64 \%$ & 77,848 & $39 \%$ & 17,995 & $60 \%$ & 125,573 \\
\hline & Particular & 17,066 & $36 \%$ & 121,398 & $61 \%$ & 12,244 & $40 \%$ & 150,708 \\
\hline & Total & 46,796 & $100 \%$ & 199,246 & $100 \%$ & 30,239 & $100 \%$ & 276,281 \\
\hline \multirow{3}{*}{ 2012-2013 } & Público & 29,966 & $63 \%$ & 81,655 & $40 \%$ & 18,587 & $58 \%$ & 130,208 \\
\hline & Particular & 17,798 & $37 \%$ & 121,856 & $60 \%$ & 13,425 & $42 \%$ & 153,079 \\
\hline & Total & 47,764 & $100 \%$ & 203,511 & $100 \%$ & 32,012 & $100 \%$ & 283,287 \\
\hline \multirow{3}{*}{ 2013-2014 } & Público & 30,753 & $61 \%$ & 80,702 & $39 \%$ & 20,384 & $56 \%$ & 131,839 \\
\hline & Particular & 19,483 & $39 \%$ & 127,560 & $61 \%$ & 15,702 & $44 \%$ & 162,745 \\
\hline & Total & 50,236 & $100 \%$ & 208,262 & $100 \%$ & 36,086 & $100 \%$ & 294,584 \\
\hline \multirow{3}{*}{ 2014-2015 } & Público & 31,250 & $60 \%$ & 82,635 & $37 \%$ & 22,550 & $58 \%$ & 136,435 \\
\hline & Particular & 21,228 & $40 \%$ & 139,745 & $63 \%$ & 16,589 & $42 \%$ & 177,562 \\
\hline & Total & 52,478 & $100 \%$ & 222,380 & $100 \%$ & 39,139 & $100 \%$ & 313,997 \\
\hline \multirow{3}{*}{$2015-2016$} & Público & 31,204 & $60 \%$ & 83,716 & $35 \%$ & 23,282 & $60 \%$ & 138,202 \\
\hline & Particular & 21,125 & $40 \%$ & 153,615 & $65 \%$ & 15,488 & $40 \%$ & 190,228 \\
\hline & Total & 52,329 & $100 \%$ & 237,331 & $100 \%$ & 38,770 & $100 \%$ & 328,430 \\
\hline \multirow{3}{*}{ 2016-2017 } & Público & 32,352 & $58 \%$ & 83,364 & $35 \%$ & 23,888 & $61 \%$ & 139,604 \\
\hline & Particular & 22,913 & $42 \%$ & 156,032 & $65 \%$ & 15,560 & $39 \%$ & 194,505 \\
\hline & Total & 55,625 & $100 \%$ & 239,396 & $100 \%$ & 39,448 & $100 \%$ & 334,109 \\
\hline \multirow{3}{*}{ 2017-2018 } & Público & 32,334 & $56 \%$ & 82,981 & $33 \%$ & 25,095 & $57 \%$ & 140,410 \\
\hline & Particular & 25,254 & $44 \%$ & 167,619 & $67 \%$ & 18,649 & $43 \%$ & 211,522 \\
\hline & Total & 57,588 & $100 \%$ & 250,600 & $100 \%$ & 43,744 & $100 \%$ & 351,932 \\
\hline
\end{tabular}

Fuente: Elaboración propia con base en SEP, 2019. 
SALCEDA, J. P.; ALCÁNTARA, A.

Respecto a la participación de la matrícula por nivel de posgrado se puede notar que los estudios de maestría conservan el mayor porcentaje de matrícula, seguido por la especialidad y el doctorado, sin embargo, podemos notar que durante el periodo analizado la especialidad pierde participación porcentual respecto a la maestría y doctorado. La especialidad inicia con 21.4 por ciento y termina con 16 , lo que significa que pierde poco más de cinco puntos porcentuales. En cambio, la maestría y el doctorado ganan poco más de dos puntos porcentuales cada uno (ver Tabla 3).

Tabla 3. Participación porcentual por nivel de posgrado 2008-2018

\begin{tabular}{|c|c|c|c|}
\hline Ciclo & $\mathrm{E}$ & $\mathrm{M}$ & $\mathrm{D}$ \\
\hline $2008-2009$ & $21.4 \%$ & $68.6 \%$ & $10.0 \%$ \\
\hline $2009-2010$ & $20.3 \%$ & $69.1 \%$ & $10.6 \%$ \\
\hline $2010-2011$ & $19.5 \%$ & $69.4 \%$ & $11.1 \%$ \\
\hline $2011-2012$ & $16.9 \%$ & $72.1 \%$ & $10.9 \%$ \\
\hline $2012-2013$ & $17.0 \%$ & $71.8 \%$ & $11.3 \%$ \\
\hline $2013-2014$ & $17.0 \%$ & $70.7 \%$ & $12.2 \%$ \\
\hline $2014-2015$ & $17.0 \%$ & $70.8 \%$ & $12.5 \%$ \\
\hline $2015-2016$ & $16.0 \%$ & $72.3 \%$ & $11.8 \%$ \\
\hline $2016-2017$ & $17.0 \%$ & $71.7 \%$ & $11.8 \%$ \\
\hline $2017-2018$ & $16.0 \%$ & $71.2 \%$ & $12.4 \%$ \\
\hline
\end{tabular}

Fuente: Elaboración propia con base en SEP, 2019.

\section{INCREMENTO DE MATRÍCULA POR NIVEL}

Durante la década analizada el mayor incremento porcentual de matrícula lo obtuvo el doctorado del sector particular, dado que alcanzó 284 por ciento, seguido por la maestría con 132 por ciento y la especialidad con 89 por ciento. En los tres niveles de posgrado el sector público obtuvo menores incrementos porcentuales que el sector particular (ver Tabla 4).

Tabla 4. Incremento porcentual de la matrícula de posgrado por nivel y régimen de sostenimiento

\begin{tabular}{|l|r|r|r|r|r|r|}
\hline \multirow{2}{*}{ Ciclo escolar } & \multicolumn{2}{|c|}{ Especialidad } & \multicolumn{2}{c|}{ Maestría } & \multicolumn{2}{c|}{ Doctorado } \\
\cline { 2 - 7 } & Público & Particular & Público & Particular & Público & Particular \\
\hline $2008-2009$ & 26,348 & 13,356 & 54,864 & 72,192 & 13,676 & 4,854 \\
\hline $2017-2018$ & 32,344 & 25,254 & 82,981 & 167,619 & 25,095 & 18,649 \\
\hline $\begin{array}{l}\text { Incremento } \\
\text { porcentual }\end{array}$ & $23 \%$ & $89 \%$ & $51 \%$ & $132 \%$ & $83 \%$ & $284 \%$ \\
\hline
\end{tabular}

Fuente: Elaboración propia con base en SEP, 2019. 


\section{INSTITUCIONES QUE OFRECEN POSGRADOS EN MÉXICO}

En congruencia con el incremento de la matrícula podemos observar que fueron las instituciones y escuelas del sector particular las que obtuvieron el mayor incremento porcentual. Las instituciones particulares se incrementaron 175 por ciento, mientras que las escuelas alcanzaron 148 por ciento.

Tabla 5. Instituciones y escuelas que ofrecen posgrado por régimen de sostenimiento 20082018

\begin{tabular}{|l|c|c|c|c|}
\hline \multirow{2}{*}{ Ciclo } & \multicolumn{2}{|c|}{ Público } & \multicolumn{2}{c|}{ Particular } \\
\cline { 2 - 5 } & Instituciones & Escuelas & Instituciones & Escuelas \\
\hline $2008-2009$ & 244 & 836 & 591 & 817 \\
\hline $2017-2018$ & 467 & 1401 & 1623 & 2029 \\
\hline $\begin{array}{l}\text { Incremento } \\
\text { porcentual }\end{array}$ & $91 \%$ & $68 \%$ & $175 \%$ & $148 \%$ \\
\hline
\end{tabular}

Fuente: Elaboración propia con base en SEP, 2019.

El sistema de educación superior es cada vez más complejo y diverso. Un aspecto relevante para el posgrado es saber si las instituciones están formando capital humano de alto nivel que puedan dedicarse a la investigación. Un estudio muestra que el crecimiento de la educación superior privada obedece al surgimiento de instituciones "[...] de absorción de demanda" o "de bajo perfil", lo que significa que este tipo de instituciones "no se distingue por elevados estándares de calidad o una importante planta docente [...]" (SILAS, 2005, p. 3032). Juan Carlos Silas utiliza la tipología de Daniel Levy (1995) para explicar el surgimiento de instituciones de tipo superior privada, clasificándolas de la siguiente manera:

- Primera ola: Universidad Iberoamericana, Universidad Panamericana, Universidad La Salle, Universidad Anáhuac, Instituto Tecnológico y de Estudios Superiores de Occidente (Guadalajara), Universidad del Valle de Atemajac (UNIVA) (Guadalajara) y la Universidad de Monterrey.

- Segunda ola: el Instituto Tecnológico y de Estudios Superiores de Monterrey, Universidad Autónoma de Guadalajara, el Instituto Tecnológico Autónomo de México, la Universidad de las Américas-Puebla y el Sistema CETYS- Universidad en Baja California.

- Tercera ola: instituciones que tratan de absorber la demanda insatisfecha por los otros tres grupos de instituciones (públicas, católicas y de élite) (SILAS, 2005).

Aunque Silas hace una asociación entre esta tipología con el incremento de matrícula de licenciatura, tomamos en cuenta su propuesta porque consideramos que son estos mismos tipos de instituciones los que están atendiendo a la mayor parte de la matrícula de posgrado. 
SALCEDA, J. P.i ALCÁNTARA, A.

\section{LOS PROGRAMAS DE POSGRADOS DE CALIDAD}

Los programas de evaluación de la calidad de los posgrados han sido operados por el CONACYT desde 1991 hasta la fecha. A lo largo de estos años han existido tres programas: 1) el Programa de Posgrados de Excelencia (PPE) (1991-2000); 2) el Programa de Fortalecimiento al Posgrado Nacional (PFPN) (2001-2006); y el Programa Nacional de Posgrados de Calidad (PNPC) (2007-en adelante). Este programa opera bajo el método de evaluación de pares; los coordinadores de los programas organizan y analizan información de los resultados del programa de acuerdo con los marcos de referencia establecidos por el CONACYT en coordinación con la Subsecretaría de Educación Superior de la SEP, los pares asignados revisan la información y se conforma una plenaria para valorar la decisión de acreditar o no al programa. Posteriormente, el Consejo Nacional de Posgrado revisa los procesos de evaluación, si no existen inconsistencias aprueba las decisiones de los comités de pares; de lo contrario, se puede solicitar la reposición del proceso de evaluación.

El problema respecto a la evaluación de la calidad del posgrado radica en que menos del 20 por ciento de programas de la oferta total del país está acreditado. En 2008 existían 6 mil 325 programas, de los cuales solo 1,072 estaban acreditados (CONACYT, 2009); en 2012 el total de fue de 8 mil 265, pero solamente 1,583 pertenecían al PNPC (ALCÁNTARA \& DE LA CRUZ, 2015), esto significa 17 y 19 por ciento, respectivamente.

Un dato relevante para este estudio es la composición del Padrón del PNPC por sector académico porque podemos ver cuáles son las instituciones que ocupan un papel importante y cuáles son las que se han quedado atrás. Así, observamos que la Universidades Públicas Estatales (UPES) tienen la mayor participación porcentual, seguidas por las instituciones federales. Con datos del Informe General del Estado de la Ciencia y la Tecnología 2009 notamos que en 2008, el Padrón del PNPC estaba integrado por 1,072 programas de los cuales solo 6.5 por ciento pertenecía a una institución privada (CONACYT, 2010). Si comparamos estos datos con los de 2012 y 2018 podemos ver que a lo largo de la década no hubo modificaciones respecto a la participación del sector particular, dado que el porcentaje osciló entre 6 y 7, lo que puede ser una variación normal cuando sabemos que los programas pueden perder el registro durante algunos periodos.

Tabla 6. Programas con registro de calidad por sector académico 2012-2018

\begin{tabular}{|c|c|c|c|c|c|c|c|c|c|c|}
\hline \multirow{3}{*}{ Sector académico } & \multicolumn{6}{|c|}{ Nivel } & \multirow{2}{*}{\multicolumn{4}{|c|}{ Total }} \\
\hline & \multicolumn{2}{|c|}{ Especialidad } & \multicolumn{2}{|c|}{ Maestría } & \multicolumn{2}{|c|}{ Doctorado } & & & & \\
\hline & 2012 & 2018 & 2012 & 2018 & 2012 & 2018 & 2012 & $\%$ & 2018 & $\%$ \\
\hline $\begin{array}{l}\text { Instituciones de Educación } \\
\text { Superior Públicas de los } \\
\text { Estados (UPES) }\end{array}$ & 130 & 245 & 498 & 735 & 239 & 364 & 867 & 55 & 1344 & 57.3 \\
\hline $\begin{array}{l}\text { Instituciones de Educación } \\
\text { Superior Públicas } \\
\text { Federales }\end{array}$ & 12 & 105 & 159 & 186 & 103 & 118 & 274 & 17 & 409 & 17.4 \\
\hline
\end{tabular}




\begin{tabular}{|l|c|c|c|c|c|c|c|c|c|c|}
\hline Centros Conacyt & 5 & 5 & 77 & 90 & 53 & 57 & 135 & 8.5 & 152 & 6.5 \\
\hline $\begin{array}{l}\text { Instituciones de Educación } \\
\text { Superior Particulares }\end{array}$ & 10 & 33 & 73 & 75 & 31 & 41 & 117 & 7.4 & 149 & 6.4 \\
\hline $\begin{array}{l}\text { Centros de Investigación } \\
\text { Federales }\end{array}$ & 0 & 0 & 56 & 68 & 51 & 60 & 107 & 6.7 & 128 & 5.5 \\
\hline $\begin{array}{l}\text { Tecnológico Nacional de } \\
\text { México }\end{array}$ & 2 & 1 & 56 & 99 & 17 & 32 & 75 & 5 & 132 & 5.6 \\
\hline Otras instituciones & 2 & 20 & 8 & 10 & 1 & 2 & 11 & 0.7 & 32 & 1.4 \\
\hline Total & 161 & 409 & 927 & 1263 & 495 & 674 & 1583 & 100 & 2346 & 100 \\
\hline
\end{tabular}

Fuente: Elaboración propia con base en datos proporcionados por Arturo Gutiérrez, Subdirector de la Dirección de Posgrados de Conacyt.

\section{DISCUSIÓN Y CONCLUSIONES}

Nuestro objetivo fue evaluar si el posgrado en México ha crecido bajo la guía de los planes y programas de desarrollo científico y educativo. El supuesto principal fue que las políticas se diseñaron para dirigir solo al sector público del posgrado, mientras que el particular se regula por la lógica de la oferta y la demanda. Es difícil aceptar nuestro supuesto inicial porque la política científica y educativa revisada presenta distintos matices dependiendo del programa (de política científica o educativa) y del periodo.

Los resultados mostraron que efectivamente, durante 2007-2012, la política científica se orientó de manera predominante al sector público en materia de formación de recursos humanos, en cambio, el PSE del mismo periodo destinó sus objetivos a la calidad del posgrado enfatizando su interés en el posgrado de las instituciones particulares. No es casualidad que la política científica se oriente al sector público y la educativa al sector privado, en esta postura se encuentra implícita la idea de que la responsabilidad de fortalecer la ciencia corresponde al sector público, descartando al sector particular.

En México, la tradición era esperar que el sector público fuera el responsable de formar a los doctores del país porque durante una primera etapa de expansión fue éste el que llevó el mayor peso, pero no solo eso, se esperaba que estos doctores se convirtieran en científicos. Todavía podemos encontrar esta idea en el Informe General del Estado de la Ciencia y la Tecnología de 2009 cuando se señala que: "El doctorado es el nivel académico necesario para atender la esfera de competencias propias de la investigación, desarrollo tecnológico e innovación" (CONACYT, 2010, p. 48). Esto implicaba asignar dos funciones a los doctores: la formación de otros posgraduados, la investigación, el desarrollo tecnológico y la innovación, así se observa en la siguiente cita: 
SALCEDA, J. P.; ALCÁNTARA, A.

\begin{abstract}
El personal con nivel de doctorado es imprescindible para apuntalar la formación académica de los jóvenes en los diferentes niveles del posgrado, asimismo, estas competencias intelectuales son necesarias para identificar personal con las capacidades necesarias para la investigación, desarrollo tecnológico y la innovación. Los doctores son demandados en las IES del país para apoyar las asesorías y tutorías de estudiantes, así como para fortalecer los trabajos de consultoría industrial y de servicios que estas organizaciones suministran a las empresas del sector productivo. (CONACYT, 2010, p. 48).
\end{abstract}

Esta idea corresponde con el académico ideal de las políticas actuales, que esperan lograr un perfil académico dedicado a la docencia, la tutoría, consultoría y a otros servicios para el sector productivo. Se asume que cuando se hace referencia a la formación de recursos humanos altamente calificados se hace referencia a los investigadores, por eso, uno de los indicadores utilizados para medir la capacidad de investigación en un país solía ser el número de graduados de doctorado. Sin embargo, estos supuestos no se pueden sostener.

Durante la década analizada el número de graduados de doctorado se incrementó 173 por ciento considerando el sector público y particular, dado que pasó de 2 mil 554 graduados en 2008 (CONACYT, 2010) a 6 mil 970 en 2017-2018 (ANUIES, 2017). Cuando se compara a México con otros países destaca el incremento que tuvo respecto a los graduados de doctorado. Esto parece un efecto esperado de las políticas científicas y educativas, con estos datos podríamos afirmar que el posgrado ha crecido bajo la guía de los programas gubernamentales. Sin embargo, hay que preguntarse si estos graduados se formaron para insertarse a las actividades de investigación, tal como se asumía en las políticas. La respuesta es negativa. Como pudimos observar el incremento de la matrícula de doctorado se debe principalmente al sector particular, el problema es que solo un número reducido de instituciones de este sector cuenta con programas reconocidos por su calidad y, por ende, con capacidad suficiente para formar investigadores, el resto de las instituciones no cuentan con ninguna de estas cualidades.

Asimismo, habría que analizar cuáles son los campos de conocimiento en los que se formaron estos doctores, pues son las IES públicas las que ofrecen el mayor número de programas en las ciencias naturales y exactas, e ingenierías, así como en las ciencias sociales y humanísticas. Por tanto, en términos generales las IES del sector público, junto con algunas del extranjero, son las que forman a la mayor parte de los científicos o tecnólogos del país.

Con los resultados del estudio podemos decir que nuestro supuesto inicial se acepta para el análisis del PECyT 2008-2012, porque sus planes se centran en el sector público, lo que no sucede con el resto de los documentos analizados, por lo que debemos agregar otra explicación al problema. En los programas de política científica (Peciti 2014-2018) y educativa (PSE, 2007-2012 y 2013-2018) se identifican los problemas asociados al posgrado y se plantean objetivos, estrategias y acciones para combatirlos, tanto para el sector público, como particular, en estos casos, el problema no es que las políticas se centren en el sector público, 
pues como vimos el PSE de 2007 se orienta de manera predominante al sector particular, el problema es que estos programas de política no logran su implementación.

Como ya se mencionó antes, los principales problemas identificados respecto al posgrado fueron: a) la insuficiencia de recursos humanos, específicamente de graduados de doctorado, y b) incrementar los programas de posgrado con registro de calidad. Los resultados respecto a los recursos humanos ya se explicaron, ahora veamos los resultados respecto a los programas de calidad.

En los diagnósticos de los programas de política es evidente la preocupación respecto a la calidad del posgrado y los objetivos propuestos, sobre todo en el PSE 2007-2012 son congruentes con el problema, sin embargo, no se obtienen los resultados deseados. La evaluación de la calidad del posgrado en el país existe desde hace 29 años, sin embargo, la cantidad de posgrados que se evalúan y logran su acreditación son menos del 20 por ciento de la oferta total; además, este 20 por ciento, está compuesto casi en su totalidad por instituciones del sector público.

Es importante señalar que el principal problema sobre la calidad del posgrado se encuentra en las instituciones del sector particular, dado que del total de programas que integran el padrón del PNPC solo seis o siete por ciento pertenecen al sector particular. Esta es la razón principal por la que los objetivos del PSE 2007-2012 se orienta de manera enfática a este sector. El problema está identificado en el programa de política y en estudios de investigación educativa, pues desde 2013 Magdalena Fresán señalaba tres problemas entorno al RVOE: a) la escasa profesionalización de los servidores públicos responsables de la revisión de los programas; b) aprobación de programas independientemente del cumplimiento de las normas y requisitos establecidos, derivado de negociaciones políticas; $c$ ) aplicación de criterios heterogéneos a pesar de un acuerdo con normas establecidas (p. 193). El problema es que estos programas de política han pasado desapercibidos por las autoridades educativas y los Gobiernos en turno.

Artigo recebido em: 18/02/2020

Aprovado para publicação em: 30/03/2020

\section{A DECADE EVALUATING THE QUALITY OF GRADUATE PROGRAMS IN MEXICO (2008-2018)}

ABSTRACT: In this article we analyze whether graduate programs in Mexico have been developed in accordance with the objectives set in the national policy programs during the 2008-2018 period. Using the evaluative research method, we contrast some objectives of the national policy programs with the increase and distribution of graduate programs, enrollment and programs accredited for their quality, distinguishing public and private sectors. The results show that the objectives of the policies sought to solve: the lack of human resources for research, and the low numbers of quality graduate programs. 
SALCEDA, J. P.; ALCÁNTARA, A.

However, statistical data analysis shows that these objectives had no effect on the development of new and better graduate programs, resulting in an accelerated increase of programs in the private sector without quality accreditation.

KEYWORDS: Graduate education. Evaluation. Quality. Mexico.

UMA DÉCADA AVALIANDO A QUALIDADE DA PÓS-GRADUAÇÃO NO MÉXICO (2008-2018)

RESUMEN: Neste artigo, analisamos se os estudos de pós-graduação no México foram desenvolvidos de acordo com os objetivos definidos nos programas de política nacional durante 2008-2018. Por meio do método de pesquisa avaliativa, contrastamos os objetivos da política com o comportamento (aumento e distribuição) das instituições, matrículas e programas acreditados por sua qualidade, distinguindo os setores público e privado. Os resultados mostram que os objetivos da política buscaram solucionar: os recursos humanos insuficientes para a pesquisa e o baixo percentual de cursos de pós-graduação de qualidade. No entanto, a análise dos dados estatísticos mostra que esses objetivos não tiveram efeito sobre o desenvolvimento da pós-graduação, resultando em um aumento acelerado da iniciativa privada em programas sem acreditação de qualidade.

PALABRAS CLAVE: Pós-graduação. Avaliação. Qualidade. México.

\section{LISTA DE REFERENCIAS}

ALCÁNTARA, A.; DE LA CRUZ, G. El posgrado en México a partir de los datos del ExECUM 2007-2012. 1a Edición. México: Universidad Nacional Autónoma de México, 2015.

ANUIES. Anuario Educación Superior Posgrado. 2017-2018. México, 2017.

AURIOL, L. Careers of Doctorate Holders: Employment and Mobility Patterns. OECD Science, Technology and Industry Working Papers, 1a. Edición. Francia, 2010.

CABRERA, F. Investigación evaluativa en la educación, en VARIOS AUTORES.Técnicas de evaluación y seguimiento de programas en Formación Profesional. Madrid: Largo Caballero, 1987.

CONACYT. Programa Especial de Ciencia y Tecnología 2008-2012. México: ANUIES, 2008.

. Capítulo II. Recursos humanos en ciencia y tecnología. En Informe General del Estado de la Ciencia y la Tecnología. México, 2009. Disponible en: http://www.siicyt.gob.mx/index.php/transparencia/informes-conacyt/informe-general-delestado-de-la-ciencia-tecnologia-e-innovacion/informe-general-2009. Acceso el: 12 feb. 2020. 
Una década evaluando la calidad del posgrado...

Dossiê 668

. Programa Especial de Ciencia, Tecnología e Innovación 2014-2018. México,

2014.

. Capítulo II. Recursos humanos en ciencia y tecnología. En Informe General del

Estado de la Ciencia y la Tecnología. México, 2017. Disponible en:

http://www.siicyt.gob.mx/index.php/transparencia/informes-conacyt/informe-general-del-

estado-de-la-ciencia-tecnologia-e-innovacion/informe-general-2017. Acceso el: 12 feb. 2020.

FRESÁN, M. Acreditación del posgrado. Institucionalización e impacto en Argentina y

México. 1a. Edición. México: Universidad Autónoma Metropolitana y ANUIES, 2013.

LEVY, D. C. La educación superior y el Estado en Latinoamérica. Retos privados al predominio público. México: FLACSO, 1995.

MENDOZA, J. Subsistemas de Educación Superior. Estadística básica 2006-2017. México: Universidad Nacional Autónoma de México, 2018.

PATIÑO, J. Implementación y efectos de las políticas de posgrado en dos universidades públicas estatales. Tesis (Doctorado en Educación) -Instituto de Ciencias de la Educación de la Universidad Autónoma del Estado de Morelos. 2013.

ROSSI, P. H.; FREEMAN, H. E. Evaluation: A Systematic Approach. Newbury Oark: Sage, 1993.

SANCHEZ, D. La evaluación plenaria del Programa Nacional de Posgrados de Calidad (PNPC). Programas de renovación. México: Conacyt, 2017.

SANDIN, E. Capítulo 7. Tradiciones en la investigación cualitativa. En Investigación cualitativa en educación. Fundamentos y tradiciones. México: McGraw-Hill Interamericana, 2003.

SEP. Programa Sectorial de Educación 2007-2012. México, 2007.

SEP. Programa Sectorial de Educación 2013-2018. México, 2013.

SEP. Sistema Interactivo de Consulta de Estadística Educativa. En Dirección General de Planeación, Programación y Estadística Educativa. Disponible en:

http://planeacion.sep.gob.mx/principalescifras/. Acceso: 20 ago. 2019.

SILAS, J. C. Realidades y tendencias en la educación superior privada mexicana. Perfiles educativos, vol. XXVII, núm. 109-110, pp. 7-37, 2005. 
SALCEDA, J. P.; ALCÁNTARA, A.

WUEST, T.; JIMÉNEZ, P. El posgrado en México. 1a. Edición. México: Universidad Nacional Autónoma de México, 1990.

Josefina Patiño Salceda: Doctora en Educación por la Universidad Autónoma del Estado de Morelos (UAEM). Realizó estancia posdoctoral en el Instituto de Investigaciones sobre la Universidad y la Educación de la Universidad Nacional Autónoma de México (IISUE-UNAM). Es candidata del Sistema Nacional de Investigadores (SNI) y profesora investigadora de la Universidad Pedagógica Nacional, CDMX Norte, Unidad 096. Ha realizado varios estudios sobre el derecho a la educación, procesos de formación y políticas de la educación superior. En específico ha estudiado los cambios que ha enfrentado la formación doctoral durante las primeras dos décadas del siglo XXI.

Orcid: http://orcid.org/0000-0003-2803-378X

E-mail: josastica@gmail.com

Armando Alcántara: Doctor en Educación (Ph. D.) por la Universidad de California, Los Ángeles (UCLA). Pertenece al Sistema Nacional de Investigadores y a la Academia Mexicana de Ciencias. Es investigador titular del Instituto de Investigaciones sobre la Universidad y la Educación (IISUE) de la Universidad Nacional Autónoma de México (UNAM) Sus intereses de investigación se enfocan a la educación superior comparada, las políticas de educación superior, ciencia y tecnología, la educación para la ciudadanía y las políticas de equidad en la educación superior.

Orcid: https://orcid.ors/0000-0003-4906-3638

E-mail: aralsantuario@gmail.com

Este periódico utiliza a licença Creative Commons Attribution 3.0, para periódicos de acesso aberto (Open Archives Iniciative - OAI). 